\title{
DO PRESENCIAL AO VIRTUAL: ANALISANDO AS CONTRADIÇÕES AO REESTRUTURAR AS PRÁTICAS PEDAGÓGICAS NA FORMAÇÃO INICIAL DE PROFESSORES DE LÍNGUA INGLESA
}

\author{
Samantha G. M. Ramos \\ Universidade Estadual de Londrina
}

\begin{abstract}
RESUMO: Este artigo relata uma investigação sobre os processos de ensino situados em ambientes informatizados de aprendizagem e constituídos por Objetos de Aprendizagem Digitais (OAD). Em 2015, um grupo de docentes da UEL iniciou a criação de OAD para compor as atividades de em um curso no Moodle explorando os conteúdos teóricos básicos para a prática do futuro professor de Inglês. Neste texto, investigo as contradições que surgiram durante a elaboração destes OAD através da Teoria da Atividade (Engestrom, 2000) contando com extratos dos encontros realizados pelos docentes envolvidos e com os próprios materiais elaborados a fim de desvendar os caminhos da inserção de OAD em processos de formação inicial de professores de línguas estrangeiras.

PALAVRAS-CHAVE: Objetos digitais, aprendizagem, formação inicial.
\end{abstract}

ABSTRACT: This article reports an investigation about the teaching processes taking place in computerized learning environments and constituted by Digital Learning Objects (DLO). In 2015, a group of UEL Professors started the creation of DLO to compose the activities of a course in Moodle exploring the basic theoretical framework for the practice of the future English teachers. In this paper, I investigate the contradictions that emerged during the elaboration of these DLO through the Theory of Activity (Engestrom, 2000), with excerpts from the meetings held by the teachers involved and with the materials elaborated in order to uncover the paths of DLO insertion in pre-service teacher education.

KEY WORDS: Learning objects, learning, pre-service education.

\section{Introdução}

A formação inicial de Professores de Línguas Estrangeiras, mas especificamente da Língua Inglesa (LI) tem sido alvo de inúmeras investigações que analisam os mais variados aspectos educacionais. Neste contexto, afloram os estudos que relacionam esta formação inicial à inserção de novas tecnologias educacionais e às tecnologias de informação e comunicação (TIC).

$\mathrm{Na}$ Universidade Estadual de Londrina (UEL), buscamos inserir o letramento digital nos programas e nas práticas das disciplinas que compõem a formação inicial do professor de Língua Inglesa. Sobre o conceitos de letramento digital, me apoio nas seguintes definições para o termo:

Letramento digital é a consciência, atitude e capacidade dos indivíduos de utilizar adequadamente as ferramentas e facilidades digitais para identificar, acessar, gerenciar, integrar, avaliar, analisar e sintetizar os recursos digitais, construir novos conhecimentos, criar expressões da mídia, e comunicar-se com outros, no contexto de situações específicas da vida, a fim de 
permitir a ação social construtiva, e para refletir sobre este processo. (Martin, 2006) ${ }^{1}$

Os novos letramentos da Internet e as outras tecnologias da informação e de comunicação incluem habilidades, estratégias e disposições necessárias para utilizar e adaptar-se com sucesso às rápidas mudanças nas informações, nas tecnologias de comunicação e nos contextos que continuamente emergem em nosso mundo e influenciam todas as áreas de nossa vida pessoal e profissional. Estes novos letramentos nos permitem usar a Internet e outras TIC (Tecnologias de Informação e Comunicação) para identificar questões importantes, localizar informações, avaliar criticamente a utilidade da informação, sintetizar informações para responder a essas perguntas, e então comunicação para responder aos outros (Leu et al., 2004, p $1572)^{2}$

As duas definições acima revelam dois importantes aspectos sobre letramento digital que tentamos desenvolver em nosso trabalho na UEL: 1) o letramento digital intencionalmente enfatiza não apenas o desenvolvimento de habilidades mas também de atitude, disposição e consciência e 2) o uso das TIC está constantemente mudando de domínio exigindo que o processo de se letrar digitalmente envolve a necessidade de ser estrategicamente suscetível e adaptável a mudanças.

Da mesma forma, buscamos inserir na formação inicial de nossos alunos atividades que se se caracterizam pela disponibilização de informações ao aluno que, diante do conteúdo, irá desenvolver individualmente suas reflexões e interpretações deslocando a ênfase do processo de ensino/aprendizagem para a tecnologia, mas mantendo o papel do professor como mediador dos conhecimentos ao avaliar tais produções e viabilizar feedbacks .

Por fim, compreendemos que não só o futuro professor de LI (discente), mas também o professor formador (docente) devem se engajar em um processo de letramento digital constante para se adequarem ao sistema educacional que a sociedade demanda.

\section{$\underline{\text { Sobre o contexto específico analisado }}$}

Em nosso curso de LETRAS/INGLÊS, as disciplinas de Estágio Supervisionado de Língua Inglesa (EST 113/EST 114) apresentam 200h de atividades cada, sendo que desta carga horária total/anual, $18 \mathrm{~h}$ são realizadas em forma de oficinas. Tais oficinas comumente se caracterizavam por encontros presenciais entre os estagiários e seus orientadores para o desenvolvimento de atividades que contemplassem os conteúdos programáticos dos

\footnotetext{
${ }^{1}$ Nossa tradução para: Digital literacy is the awareness, attitude and ability of individuals to appropriately use digital tools and facilities to identify, access, manage, integrate, evaluate, analyze and synthesize digital resources, construct new knowledge, create media expressions, and communicate with others, in the context of specific life situations, in order to enable constructive social action; and to reflect upon this process (Martin, 2006).

2 Nossa tradução para: The new literacies of the Internet and other information and communication technologies include the skills, strategies, and dispositions necessary to successfully use and adapt to the rapidly changing information and communication technologies and contexts that continuously emerge in our world and influence all areas of our personal and professional lives. These new literacies allow us to use the Internet and other ICTs to identify important questions, locate information, critically evaluate the usefullness of the information, synthesize information to answer those questions, and then communication to answer to others (Leu et al. 2004, p 1572).
} 
respectivos programas (pressupostos teóricos sobre processos de ensino/aprendizagem de Línguas Estrangeiras $\left.{ }^{3}\right)$.

De fato, em 2005, professoras formadoras (mais especificamente aquelas engajadas na orientação de estágio) se reuniram para a criação de um material a ser construído colaborativamente para ser utilizado nas chamadas oficinas de estágio. O material impresso, nomeado ROTEIROS PEDAGÓGICOS (2008), e se tornou disponível durante os anos subsequentes pra ser implementado no contexto para o qual foi criado.

Entretanto, em 2015, três professoras formadoras (também envolvidas na orientação de estágio curricular) decidiram "transportar" estas atividades presenciais para o Moodle, um ambiente virtual de aprendizagem (AVA), a partir da criação de Objetos de Aprendizagem Digitais (OAD) que passariam a ser utilizados como novos artefatos de mediação na construção do conhecimento pretendido. A este processo de criação de $\mathrm{OAD}$, demos o nome de SAPROMO - Sistema de Atividades de Produção de Oficinas Moodle. Em suma, este texto é um relato/ análise desta decisão e de todos os conflitos que se criaram a partir desta dela.

\section{$\underline{\text { Sobre a criação de Objetos de Aprendizagem Digitais }}$}

O termo "objetos de aprendizagem" tem sido alvo de estudos educacionais e várias definições foram criadas para esclarecer sua natureza. Nesta pesquisa, optamos pela definição de Wiley (2000) ao afirmar que os OA são "quaisquer recursos digitais que possam ser reutilizados para dar suporte a aprendizagem"4 e que possam ser disponibilizados via rede de computadores podendo ser de pequeno porte (imagens digitais, feed de dados ao vivo, vídeos ao vivo ou pré-gravados, pequenos textos, animações e aplicativos) ou de grande porte (páginas da rede que combinem textos, imagens e outras mídias e aplicativos).

A partir da definição acima, conceituamos os OAD como recursos que podem ser elaborados pelos próprios professores através da fragmentação dos conteúdos a serem ministrados, sendo que uma vez elaborados, estes podem ser reutilizados em outros momentos, outros contextos e com outros aprendizes. Entendemos que qualquer material eletrônico que prove informações para a construção de conhecimento pode ser considerado um OAD. De acordo com Hughes at al (2009)

Os objetos de aprendizagem são unidades autônomas e distintas de materiais educativos, reunidos em torno de objetivos de aprendizagem específicos, que são utilizados para criar materiais educativos maiores, tais como aulas, módulos ou até cursos inteiros, e que seguem os requisitos de um determinado currículo de estudos. Os objetos de aprendizagem variam em tamanho, dimensão, conteúdo e aplicação, e são criados de forma a poderem existir isoladamente, serem reutilizados e ir ao encontro das exigências dos alunos. (Hughes et al, 2009, p.75)

Ainda segundo Hughes et al (2009), no que tange as características dos OA, podem-se destacar as seguintes potencialidades: a) reutilização - conteúdos de aprendizagem divididos em pequenas unidades de ensino, passíveis de serem agrupados e reagrupados em vários

\footnotetext{
${ }^{3}$ Diários de observação e regência, gerenciamento de sala de aula, planejamento de cursos/ aulas, avaliação da aprendizagem, produção e compreensão oral, produção e compreensão escrita, entre outros temas.

4 Nossa tradução para: as "any digital resource that can be reused to support learning."
} 
cursos, b) compatibilidade - unidades de ensino compatíveis, independentemente do seu criador ou do sistema de gestão de aprendizagem utilizado, c) durabilidade - unidades de ensino que resistem à evolução e à apresentação de novas tecnologias, sem se tornarem obsoletas, d) acessibilidade - conteúdo de aprendizagem que está disponível a qualquer hora e em qualquer lugar, podendo ser encontrado e reutilizado através de diferentes redes.

No processo de criação dos OA, Hughes et al(2009) sugere considerar os seguintes aspectos: a) o público: refletindo sobre a que tipo de alunos se destina o OA, b) o objetivo: definindo quais são objetivos de aprendizagem e qual o benefício que os alunos vão retirar, c) A motivação: avaliando qual o valor que o OA trará para a experiência pedagógica e d) o design - atentar para a forma na qual os alunos vão envolver-se ou interagir com o material. No que se refere a este último item, o design, os autores afirmam que:

Desenhar o 'percurso para a aprendizagem' é não só o primeiro passo como também o mais importante. Trata-se de um mapa daquilo que o aprendente vai experienciar e dos passos que terá de seguir até alcançar o objetivo da aprendizagem. Caso esteja familiarizado com a ideia, um fluxograma pode ser-lhe bastante útil. Caso contrário, pode utilizar um software para mapas mentais (mind-mapping software). Uma boa opção é, por exemplo, o Personal Brain, que contrariamente a muitos softwares do género, permite-lhe adicionar hiperligações e conteúdos ao seu 'mapa'. Ou então, pode rabiscar num pedaço de papel os pontos principais e definir qual a será a sua sequência (...) $O$ percurso para a aprendizagem deve incluir materiais, tarefas e atividades, bem como diferentes formas de apresentar a informação que, por sua vez, deverão respeitar diferentes estilos de aprendizagem ou preferências. É possível alcançar os mesmos resultados de aprendizagem seguindo caminhos diferentes. (Hughes et al, 2009, p. 78)

Ainda sobre o percurso da aprendizagem, os autores apontam que a maioria das pessoas está habituada a pensar de forma sequencial, entretanto, este tipo de planificação pode não funcionar com e-learning. Desta forma, ao projetar um OA, o professor deve ter em mente que: a) os textos a serem disponibilizados obterão melhor resultado quando são escritos em 'pedaços', que podem ser consultados como e quando o aluno quiser; b) imagens, som e vídeo devem ser incluídos ao clicar em pontos de acesso à Internet sem fios; c) menus e tabulações permitem ao aluno fazer escolhas e optar por diferentes níveis; d) páginas da Web podem ser ordenadas de modo a que pedaços de texto e imagens possam existir de forma autónoma e isolada e, por fim, e) hiperligações permitem ao utilizador procurar ou explorar tópicos relacionados (Hughes et al, 2009, p. 79).

A partir desta breve descrição do contexto e dos pressupostos teóricos elencados, prossigo o relato/análise do SAPROMO e o processo de criação dos OAD para as oficinas moodle com o intuito de caracterizar estas ações como um sistema de atividades. Durante esta investigação me apoio nos seguintes questionamentos: 1) Como passa a ser caracterizado o processo de ensino/aprendizagem das oficinas teóricas? 2) Que conflitos emergiram da criação de OAD pelas docentes/orientadoras de estágio? 
Em busca de respostas a estes questionamentos utilizarei a Teoria da Atividade como pressuposto teórico/metodológico utilizando as transcrições de três encontros presenciais e diversas mensagens trocadas no facegroup criado para facilitar a comunicação e a realização das ações. Em seguida, tecerei algumas conclusões sobre como se deu a reestruturação das práticas pedagógicas na formação inicial de Professores de Língua Inglesa no contexto aqui relatado. Em outras palavras, pretendo revelar os caminhos tortuosos que as docentes percorreram ao abandonar a utilização de um material impresso pré-existente e se enveredarem pelas trilhas tortuosas da criação de materiais para ensino a distância (EAD).

\section{Metodologia}

A premissa central da teoria da atividade é que quando os sujeitos se engajam em ações conjuntas ou em ações mediadas por artefatos ou ferramentas culturalmente construídas (tendo em mente o objeto - as metas para quais as atividades são direconadas), estas ferramentas mudam a referida atividade e as condições que a influenciam.

A terceira geração da teoria da atividade (doravante TA) de Engeström (1999) está situada nas teorias socioculturais que, por sua vez, podem ser usadas para analisar as complexidades das atividades acadêmicas tais como a criação de OAD para processos de aprendizagem mediados por tecnologia. Esta teoria também apresenta um quadro para analisar influências socioculturais sobre regras e normas, comunidades e divisão de trabalho no mesmo sistema de atividades. De fato, a análise da TA descreve estes componentes e examina suas interrelações para identificar affordances ${ }^{5}$ e contradições no sistema. Por fim, um sistema de atividade ativo não é independente de outros sistemas e uma análise deve considerar sistemas de atividades correlacionados. Qualquer contradição pode ser potencialmente endereçada transformando a atividade e alcançando resultados aprimorados.

Como mencionado anteriormente, a unidade básica de análise neste texto é o SAPROMO (Sistema de Atividades de Produção de Oficinas Moodle) com foco no conjunto de ações desenvolvidas pelas professoras formadoras em 2015 com o intuito de alterar as regras préestabelecidas e criar novos artefatos mediadores para os conteúdos teóricos das disciplinas EST113 e 114. A seguir descrevo cada um dos componentes deste sistema antes de considerar as relações de seus componentes e os outros sistemas de atividades relacionados.

No que concerne aos sujeitos da atividade, as três professoras formadoras (todas com mais de quinze anos de experiência na formação de professores de inglês em contextos universitários) $^{6}$ engajaram-se voluntariamente na criação de OAD para viabilizar os conteúdos teóricos em formato digital e em ambiente virtual de aprendizagem. Duas destas professoras já coordenam projetos de pesquisa relacionados à inserção de TICs no processo educativo e a terceira diz ter urgência em se "ambientar" nestes contextos informativos em virtude das exigências da profissão.

\footnotetext{
${ }^{5}$ Entendemos que "affordance" é o potencial de um objeto de ser usado como foi projetado para ser usado.

${ }^{6}$ As três docentes já haviam trabalhado como orientadoras de estágio e cumprido os conteúdos teóricos exigidos de forma presencial e com a utilização do material produzidos em 2005.
} 
Entendo que os artefatos se caracterizam pelo "local" onde as mudanças são possíveis, e que, se quisermos mudar a maneira que os alunos aprendem, temos que mudar os artefatos que professores e alunos utilizam. Com isto em mente, a construção de novos artefatos mediadores para as oficinas teóricas foi uma ação definida pelos sujeitos em busca de reestruturação de todo o sistema da atividade de oficinas. De fato, o processo de criação dos artefatos foi iniciado com a exposição de uma das professoras formadoras sobre sua experiência como aluna em um curso no moodle ${ }^{7}$. A terminologia $\mathrm{OAD}$ veio à tona em um dos encontros, pois a ideia inicial era exatamente criar recursos digitais que pudessem ser reutilizados para dar suporte à aprendizagem daqueles conteúdos específicos. A escolha do moodle como ambiente virtual de aprendizagem no SAPROMO se deu por este já ser institucionalizado (http://moodleg.uel.br/).

No que refere ao objeto/ meta/ motivo para as ações, compreendo que a motivação do sujeito está na transformação do objeto em um resultado com a ajuda de diversas ferramentas/artefatos. Ainda sobre estes componentes da TA, Leontiev (1974, p.22) afirma que "o conhecimento do objeto almejado é fundamental para a boa compreensão da atividade sendo que ele a orienta, a motiva e a distingue das demais atividades". No SAPROMO, a meta é a consolidação de pressupostos teóricos sobre processos de ensino/aprendizagem de Línguas Estrangeiras e o resultado esperado é que neste processo a formação dos futuros professores seja pautada na criticidade e contemple aspectos de letramento digital. Vale ressaltar que entre os motivos para o engajamento das professoras formadoras nesta atividade está a necessidade de aprimoramento profissional das mesmas através de experiências especificas novas tecnologias, produção de materiais em EAD e coleta de dados para produção artigos científicos.

Esta comunidade, na qual o SAPROMO está inserido, é composta por professores formadores (docentes) e por futuros professores (discentes) que partilham da meta de um formação inicial voltada a criticidade e às potencialidades digitais. Esta comunidade tem suas regras estabelecidas (mediante os documentos oficiais sobre formação docente, o projeto político pedagógico do curso e os conteúdos e métodos de avaliação propostos nos programas das disciplinas) e também tem critérios para a divisão de trabalho (estabelecendo o papel dos docentes, dos membros do colegiado, dos chefes de departamento, entre outros). Dentro desta grande comunidade acadêmica, as três professoras formadoras se uniram para articular uma nova proposta e coloca-la em prática em regime experimental de forma a não infringir as regras e a divisão de trabalho tradicionalmente vivenciadas neste contexto.

Nos primeiros encontros das professoras formadoras, foram discutidas as características dos OAD a serem elaborados e estabeleceu-se que estas deveriam ser no formato de "apostilas digitais" com uma "roupagem" de material EAD, isto é, contendo hiperlinks que abririam possibilidades de acesso a textos, vídeos, entrevistas, slides entre outros recursos disponíveis na internet (Regra 1). Definido o gênero "apostila digital", iniciou-se uma discussão sobre as temáticas e conteúdos a serem explorados e, desta vez, optou-se por retomar os ROTEIROS PEDAGÓGICOS (2005) em uma tentativa de "adaptar" seus conteúdos a nova proposta

\footnotetext{
${ }^{7} \mathrm{Em}$ um dos encontros gravados, a professora formadora descreve as características dos OAD que constavam do curso que participou: apostila com tópicos do curso, power point apresentando os mesmos conteúdos da apostila em tópicos e vídeo-aula também apresentando o mesmo conteúdo da apostila mas, desta vez, com a participação assíncrona de uma professora.
} 
(Regra 2). Definido os temas, as professoras formadoras também decidiram que deveriam ser elaboradas "tarefas" referentes aos conteúdos das apostilas (para avaliar o progresso dos alunos) considerando as possibilidades de recursos oferecidas pelo próprio ambiente moodle ${ }^{8}$ (Regra 3). Da mesma forma, definiu-se que cada professora formadora ficaria responsável por elaborar uma versão preliminar das apostilas digitais e compartilhar seus resultados para a apreciação das outras professoras formadoras via facegroup objetivando um processo de construção colaborativo destes novos artefatos mediadores (Divisão de trabalho).

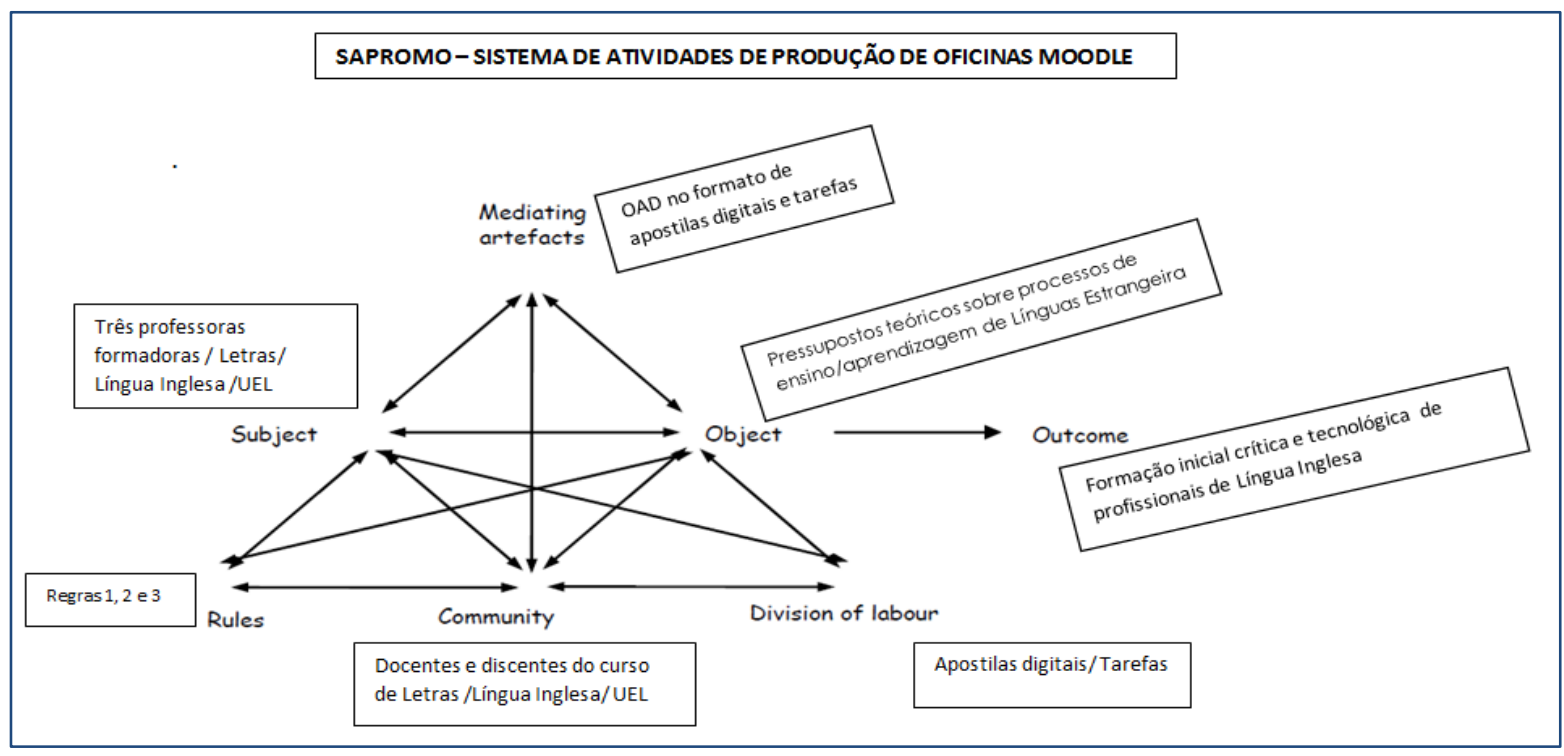

Figura 1: Representação do SAPROMO como um sistema de atividade

Segundo Roth (2004), a natureza dinâmica do sistema de atividades é central na aplicação da TA sendo que as mudanças trazem as contradições ou dilemas, distúrbios e discordâncias (Roth, 2004, p.5). As contradições podem ocorrer dentro dos componentes do sistema de atividade e/ou entre sistemas de atividades "vizinhos". No processo de criação dos OAD do SAPROMO identificamos as seguintes contradições:

Contradição 1 - Tentativa de adaptar os recursos prévios (ROTEIROS PEDAGÓGICOS) na elaboração de OAD (apostilas digitais)

Como geralmente acontece nas primeiras tentativas de produção de materiais para ambientes virtuais, as três professoras formadoras optaram por adaptar os recursos utilizados presencialmente a esta nova proposta online. Entretanto, já no início da produção das apostilas digitais, criaram-se discussões a respeito desta escolha.

O impasse é que os ROTEIROS PEDAGÓGICOS (2005) haviam sido criados objetivando a formação inicial de professores reflexivos sendo que esta se daria a partir dos questionamentos presentes no referido instrumento e mediante a natureza das interações presenciais que supostamente ocorreriam a partir destes questionamentos. Entre outras palavras, a ênfase do material era incitar debates nos quais o aprendizado ocorreria de forma colaborativa. Por sua vez, as apostilas digitais do SAPROMO vislumbram um aluno

\footnotetext{
${ }^{8}$ O sistema moodle oferece os seguintes recursos para armazenamento e avaliação de tarefas: base de dados, chats, fórum, glossário, laboratório de avaliação, lição, pesquisa de avaliação, questionário, tarefa, wiki, arquivo, conteúdo do pacote, livro, página, pasta, rótulo e url.
} 
autônomo que passa a interagir com a apostila e os hiperlinks (textos, vídeos, entrevistas, slides, etc) sendo que a construção do conhecimento se dará pela mediação da própria apostila digital e a realização das tarefas.

Em suma, a REGRA 1 estabelecida pelas professoras formadoras se confirmou inviável devido a natureza das propostas subjacentes a cada um dos materiais. A contradição/tensão 1, portanto, se encontra entre os sujeitos e as regras pré-definidas. Para superar esta contradição, os ROTEIROS PEDAGÓGICOS (2005) passaram e definir apenas as temáticas das apostilas pedagógicas e não mais a natureza das atividades.

Contradição 2 - As peculiaridades dos materiais digitais e suas tarefas

$\mathrm{Na}$ prática, a produção colaborativa que se almejava encontrou desafios para sua concretização. As professoras formadoras assumiam pela primeira vez o desafio de elaborar materiais para ambientes virtuais sendo que estas desconheciam suas características.

Em suma, esta tensão se estabeleceu entre os sujeitos e os próprios artefatos e, para superar esta contradição, foi necessário que as professoras formadoras se engajassem em um processo de estudo sobre as características de materiais digitais de modo a se tornar conscientes das demandas desta atividade.

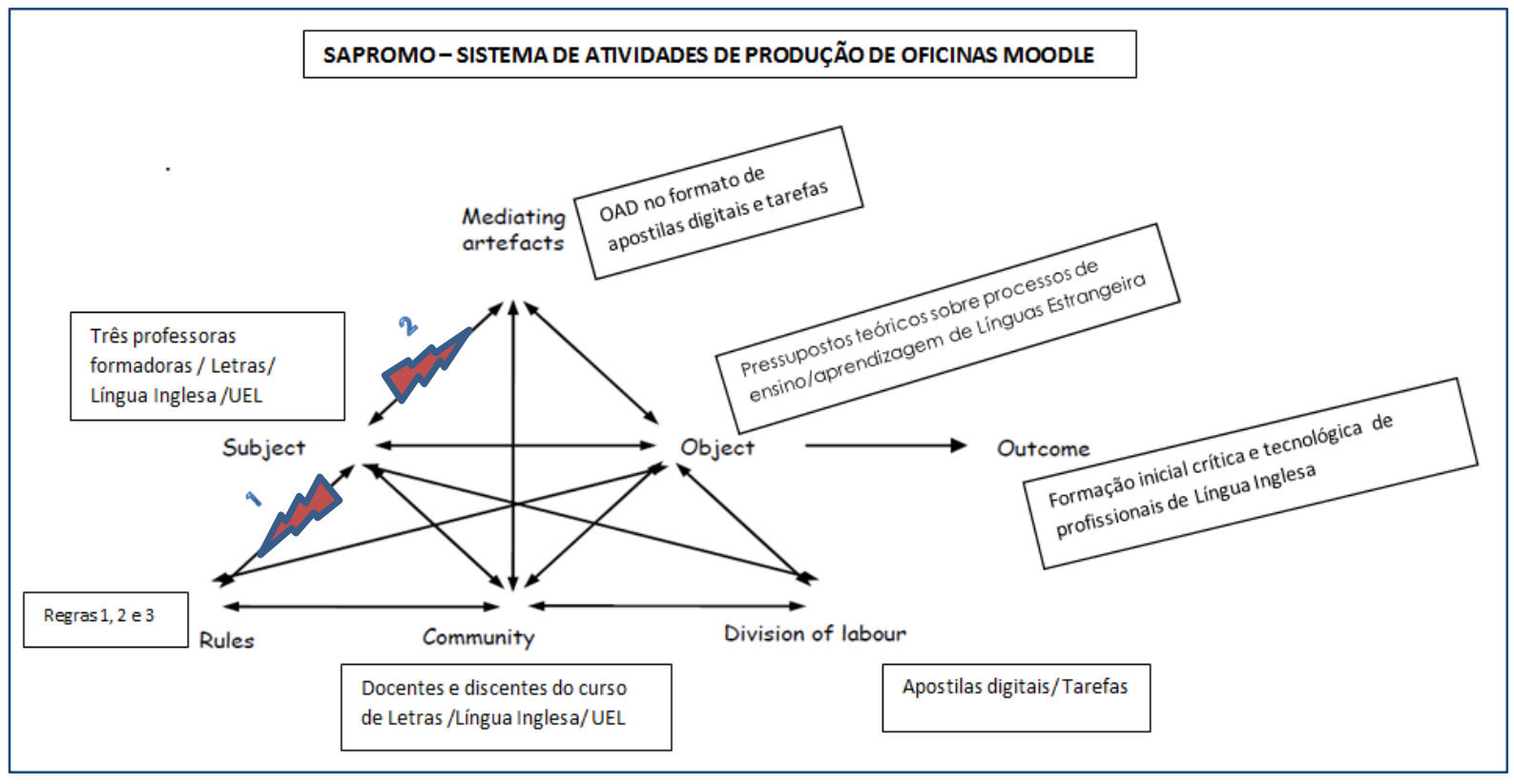

Figura 2: Representação do SAPROMO como um sistema de atividade/ Contradições 1 e 2

\section{Resultados}

Como mencionado anteriormente, o engajamento das professoras formadoras no SAPROMO em 2015 foi motivado pela urgência de promoção do letramento digital entre os futuros professores. Para isto elas julgaram necessário criar novos artefatos e estabelecer novas regras para as 18h de oficinas teóricas das disciplinas EST113 e 114. 
Sobre a nova caracterização o processo de ensino/aprendizagem das oficinas teóricas, entendo que o processo de criação das apostilas digitais de fato abre espaço para novas possibilidades de aquisição do conhecimento ao exigir do futuro professor autonomia e competência digital.

Sobre os conflitos/ contradições/tensões que emergiram da criação das apostilas digitais apontei que a proposta de adaptar os recursos prévios (ROTEIROS PEDAGÓGICOS) na elaboração dos OAD se mostrou ineficaz diante das diferenças entre a natureza das interações que se estabelecem em contextos presenciais e virtuais. Da mesma forma, apontei que as professoras formadoras desconheciam as peculiaridades dos materiais digitais e suas tarefas e que foi preciso inserir-se em um processo de aprendizado par a realização desta atividade.

\section{Conclusões}

As possibilidades de uso do computador como ferramenta educacional está crescendo e os limites dessa expansão são desconhecidos. Cada dia surgem novas maneiras de usar o computador como recurso para enriquecer e favorecer o processo de aprendizagem. Isso nos mostra que é possível alterar o paradigma educacional; hoje centrado no ensino, para algo centrado na aprendizagem. VALENTE (1993, p.15)

As palavras de Valente (1993) são significativas para compreender que este é o momento de nos confrontarmos com novos paradigmas educacionais e reavaliar questões pertinentes aos processos de ensino, aprendizagem e letramento. Diante das novas possibilidades educacionais disponibilizadas pelas novas tecnologias, entendemos que é crucial definir os papéis que os participantes devem desempenhar nestes novos contextos que aliam ensino/aprendizagem/letramento e novas tecnologias. As professoras formadoras engajadas no SAPROMO aceitaram este desafio e promoveram mudanças em seus contextos educacionais.

Neste texto, meu objetivo foi analisar/relatar o SAPROMO caracterizando-o como um sistema de atividade focando apenas nas contradições/ tensões estabelecidas durante $o$ processo de produção das apostilas digitais. Pretendo continuar minhas análises do SAPROMO considerando outros momentos/aspectos, entre eles: a utilização dos recursos do moodle para a criação das tarefas pelas professoras formadoras, a interação dos futuros professores com o novo artefato, a construção do conhecimento na realização das tarefas entre outros. Mas estes temas para novos textos.

\section{Referências Bibliográficas:}


ENGESTRÖM, Y., Miettinen, R., \& Punamäki, R-L. (Eds.). (1999). Perspectives on activity theory. Cambridge: Cambridge University Press.

HUGUES et al (Eds.). Apoio a professores para a criação de conteúdos em ambientes de aprendizagem: Manual de e-learning para professores. Projeto TACCLE, 2009. Disponível em http://erte.dge.mec.pt/files/@crie/1283778458_TACCLEportugees.pdf Acessado em 15 de abril de 2015.

LEONTIEV, A. N. The problem of activity in psychology. Soviet Psychology, v.13, n.2, p.433, 1974.

MARTIN, A. Literacies for the digital age. In: Digital Literacies for learning, ed Allan Martin and Dan Madigan, 3-25. London: Facet Publishing, 2006

ORTENZI, D.I.B.G. et al. Roteiros Pedagógicos para a Prática de Ensino de Inglês. Londrina, EDUEL, 2008.

ROTH, W.-M. (2004). Activity theory and education: An introduction. Mind, Culture and Activity, 11, 1-8.

WILEY, D. A. (2000). Connecting learning objects to instructional design theory: A definition, a metaphor, and a taxonomy. In D. A. Wiley (Ed.), The Instructional Use of Learning Objects: Online Version. Disponível em http://reusability.org/read/chapters/wiley.doc Acessado em 15 de abril de 2015. 